Itinéraires Itinéraires

Littérature, textes, cultures

\title{
Identité générique et identité sexuelle dans « Eugénie de Franval »
}

Dominique Hölzle

\section{(2) OpenEdition}

\section{Journals}

Édition électronique

URL : http://journals.openedition.org/itineraires/736

DOI : 10.4000/itineraires.736

ISSN : 2427-920X

Éditeur

Pléiade

\section{Édition imprimée}

Date de publication : 1 mars 2014

Pagination : 141-152

ISBN : 978-2-343-02712-8

ISSN : 2100-1340

\section{Référence électronique}

Dominique Hölzle, « Identité générique et identité sexuelle dans « Eugénie de Franval » », Itinéraires [En ligne], 2013-2 | 2014, mis en ligne le 19 septembre 2014, consulté le 01 mai 2019. URL : http:// journals.openedition.org/itineraires/736 ; DOI : 10.4000/itineraires.736

\section{(C) $(\oplus \Theta$}

Itinéraires est mis à disposition selon les termes de la licence Creative Commons Attribution - Pas d'Utilisation Commerciale - Pas de Modification 4.0 International. 


\title{
Identité générique et identité sexuelle dans « Eugénie de Franval »
}

\section{Résumé}

Dans son «Idée sur les romans », Sade imagine une généalogie du genre romanesque, et distingue deux traditions : la première a à voir avec l'énergie du sublime, et est clairement placée du côté du masculin; la seconde est tendre et délicate, et est associée au féminin. Dans «Eugénie de Franval », Sade s'emploie à fusionner ces deux traditions. Une lecture de la nouvelle de Sade à la lumière de la Recherche philosophique sur l'origine de nos idées du sublime et du beau d'Edmund Burke peut nous aider à comprendre la confusion des poétiques et des identités sexuelles qui fonde le travail d'écriture du marquis.

Mots clés : Sade, Burke, histoire du roman, poétique, sublime

\begin{abstract}
In "Idée sur les romans," Sade imagines a genealogy of the novel, and distinguishes two traditions: the first one has to do with the energy of the sublime, and is clearly associated with masculine values; the second one is delicate and tender, and is linked to feminine values. In "Eugénie de Franval," Sade tries to merge these two traditions. A reading of Sade's short story in the light of Edmund Burke's A Philosophical Enquiry into the Origin of our Ideas of the Sublime and Beautiful can help us understand the confusion of poetics and of sexual identities that is at the centre of the act of writing for the marquis.
\end{abstract}

Keywords: Sade, Burke, history of the novel, poetics, sublime

Dans son « Idée sur les romans » (1800), Sade établit une généalogie du genre romanesque en distinguant deux traditions, l'une sacrée et héroïque, placée clairement du côté du masculin, l'autre délicate et tendre, associée au féminin. Significativement, cette distinction reprend les concepts esthétiques développés par Edmund Burke dans sa Recherche philosophique sur l'origine de nos idées du sublime et du beau (1757). Une lecture des Crimes de l'amour (1800), et plus particulièrement d'«Eugénie de 
Franval », au regard de cette "théorie des genres » permet de saisir la poétique de la confusion au cœur de l'écriture exotérique du marquis. Tout se passe comme si la recherche d'une forme d'écriture acceptable pour le public passait par une « féminisation » de son inspiration, à travers l'intégration dans le modèle de la nouvelle tragique d'éléments caractéristiques de la poétique galante. Cette tentative d'hybridation est cependant bien vite déjouée par l'imagination sadienne, qui, toute à sa recherche de l'énergie du sublime, organise un retour du principe masculin. Cette tension entre la tentation d'une absorption dans le féminin et la recherche d'une énergie caractéristique du masculin permet d'éclairer le personnage de Franval, et, in fine, de comprendre son échec comme créateur.

Au début de son "Idée », Sade affirme que le roman est d'abord épique, et naît avec le sentiment du sacré : «N'en doutons point : ce fut dans les contrées, qui, les premières, reconnurent des Dieux, que les romans prirent leur source $[\ldots]$ ». Dès lors, « la fiction s'empare de l'esprit des hommes ${ }^{1} »$. Tant que les sociétés sont dominées par les prêtres, les personnages mis en scène dans ces récits sont des Dieux, mais dans un mouvement progressif de sécularisation, ceux-ci laissent la place aux héros épiques. S'interrogeant sur les causes du goût de l'homme pour les romans, Sade les trouve dans ses « deux faiblesses » majeures : «Partout, il faut qu'il prie, partout, il faut qu'il aime; et voilà la base de tous les romans; il en a fait pour peindre les êtres qu'il implorait, il en a fait pour célébrer ceux qu'il aimait ${ }^{2}$ ». Les récits inspirés par le sentiment religieux, y compris les récits bibliques, sont « dictés par la terreur ou l'espoir, [et sont] sombres, gigantesques, pleins de mensonges et de fictions ${ }^{3} \gg$. L'expérience du lecteur de cette catégorie de textes a manifestement à voir avec l'expérience du sublime telle qu'elle est décrite par Burke :

Tout ce qui est propre à susciter d'une manière quelconque les idées de douleur et de danger, c'est-à-dire tout ce qui est d'une certaine manière terrible, tout ce qui traite d'objets terribles ou agit de façon analogue à la terreur, est source du sublime, c'est-à-dire capable de produire la plus forte émotion que l'esprit soit capable de ressentir ${ }^{4}$.

La «terreur » qui dicte les récits dont parle Sade est ainsi pour Burke «le principe qui gouverne le sublime ${ }^{5}$ ». Parce que cette terreur naît de la douleur, et que «les idées de douleur sont beaucoup plus puissantes

1. Sade, «Idée sur les romans », dans Les Crimes de l'amour, éd. Michel Delon, Paris, Gallimard, coll. « Folio », 1987, p. 28.

2. Ibid.

3. Ibid., p. 30.

4. Edmund Burke, Recherche philosophique sur l'origine de nos idées du sublime et du beau [1757], éd. et trad. Baldine Saint-Girons, Paris, Vrin, 2009, p. 96.

5. Ibid., p. 121. 


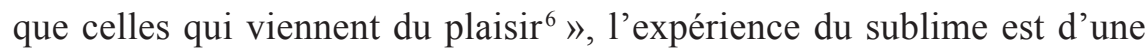
intensité incomparable. Une distance entre l'objet artistique et celui qui en jouit est cependant nécessaire. Burke l'affirme : « lorsque le danger ou la douleur serrent de trop près, ils ne peuvent donner aucun délice et sont simplement terribles; mais, à distance, et avec certaines modifications, ils peuvent être délicieux ${ }^{7}[\ldots] »$.

À la veine romanesque épique et sublime, Sade oppose dans son " Idée » une veine « sentimentale », avec des récits inspirés par l'amour, plus réels, « remplis de délicatesse et de sentiments ${ }^{8}$ », comme les romans grecs d'Héliodore, ou le Daphnis et Chloé de Longus. Nous sommes ici loin du sublime caractéristique de la première veine, et l'expérience du lecteur est alors proche de l'expérience de la «beauté » décrite par Burke. Ce dernier souligne que, pour être beau, un objet doit avoir une « apparence de délicatesse et même de fragilité ${ }^{9}$ », et il indique que « le beau se fonde sur un plaisir simple et positif et excite dans l'âme ce sentiment qu'on appelle l'amour ${ }^{10}$ ». Pleins « de goûts et d'agréables fictions » selon Sade, les romans grecs contrastent heureusement avec les romans médiévaux, « ouvrages longs, ennuyeux, empestés de superstition ${ }^{11}$ » qui proposent au lecteur un sublime lié à des terreurs métaphysiques, condamnable donc. L'Astrée d'Honoré d'Urfé, en prenant acte de la «face nouvelle» de la galanterie, réussit à rompre avec la « barbarie » et la « grossièreté ${ }^{12}$ » des romans des $\mathrm{XI}^{\mathrm{e}}$ et $\mathrm{XIII}^{\mathrm{e}}$ siècles et marque un heureux retour à l'inspiration sentimentale. Les nombreux imitateurs du texte de d'Urfé - Gomberville, La Calprenède... - le trahissent cependant en «mettant des princes ou des rois à la place des bergers du Lignon ${ }^{13} \gg$. Se trouve ainsi condamnée l'intégration de héros épiques dans des récits galants, car elle aboutit à un affadissement des modèles historiques. Les œuvres de Madame de La Fayette, plus concises et plus sobres que celles des romanciers qui l'ont précédée, en sont d'autant plus intéressantes. En expliquant que, « parce qu'elle était femme », on a prétendu que la romancière «n'avait fait ses romans qu'avec le secours de La Rochefoucauld pour les pensées, et de Segrais pour le style ${ }^{14}$ », Sade est amené à s'interroger sur les affinités entre la féminité et l'écriture romanesque, lorsqu'il s'indigne de la misogynie qu'impliquent de telles rumeurs : " Comme si ce sexe, naturellement plus délicat, plus fait pour écrire le roman, ne pouvait, en ce genre, prétendre à bien plus de
6. Ibid., p. 96.
7. Ibid., p. 97.
8. Sade, «Idée sur les romans », op. cit., p. 30.
9. Edmund Burke, op. cit., p. 202.
10. Ibid., p. 258.
11. Sade, "Idée sur les romans », op. cit., p. 32.
12. Ibid., p. 32.
13. Ibid., p. 33.
14. Ibid., p. 35. 
lauriers que nous ${ }^{15} »$. L'amour doit être le sujet central du roman, et les femmes sont les plus aptes à en parler, en témoigne la liste qu'il propose de « femmes aimables qui donnaient en ce genre de si bonnes leçons aux hommes ${ }^{16}$ ». Sont ainsi citées des romancières comme Mme de Gomez, $\mathrm{M}^{\text {lle }}$ de Lussan, Mme de Tencin, Mme de Graffigny, Mme Élie de Beaumont ou Mme Riccoboni. Mais, dans un siècle "perverti par le Régent»et marqué par l' " épicuréisme », le « ton des romans ${ }^{17}$ » s'est trouvé changé, si bien que des auteurs libertins comme Crébillon ont perverti le modèle légué par les romancières galantes. Pour Sade, le salut vient de Rousseau et du roman anglais. Il reconnaît la « délicatesse » et le « sentiment » de l'auteur de la Nouvelle Héloïse, mais c'est surtout la « vigueur » et « l'énergie » qui l'impressionnent dans ce " livre sublime ${ }^{18}$ ». L'emploi du terme « sublime » est révélateur, et pourrait désigner un ensemble romanesque qui englobe aussi les « vigoureux ouvrages » de Richardson ou de Fielding, qui tracent « des caractères mâles ${ }^{19}$ » aux prises avec l'amour. Ces auteurs nous font voir l'homme «tel qu'il peut être, tel que doivent le rendre les modifications du vice, et toutes les secousses de la passion ${ }^{20} \gg$. C'est une démesure purement humaine - et non les terreurs superstitieuses que condamnait Sade dans les romans médiévaux - qui fonde l'expérience du sublime pour le lecteur.

Il y a pour le Sade de l'« Idée » deux manières romanesques de traiter de l'amour. La manière galante ou sentimentale, perfectionnée par des romancières, examine avec délicatesse tous les raffinements du sentiment, et met le plus souvent en scène des hérö̈nes féminines. Mais il y a aussi une manière qu'on pourrait qualifier de « sublime », qui consiste à montrer les ravages de la passion et du vice dans le cœur de l'homme en convoquant des spectacles terribles et impressionnants, manière significativement illustrée principalement - mais pas exclusivement - par des auteurs masculins ${ }^{21}$, puisque Sade considère que Prévost ${ }^{22}$ ou Baculard d'Arnaud ont su trouver cette énergie du sublime dans leurs romans. Il existe une affinité entre le sexe des écrivains et le genre qu'ils pratiquent, et c'est peut-être parce qu'ils ont écrit des « romans galants », genre plus spécifiquement féminin, que Marivaux, Crébillon ou Dorat ne trouvent pas grâce aux yeux de l'auteur
15. Ibid., p. 35 .
16. Ibid., p. 36.
17. Ibid.
18. Ibid., p. 37.
19. Ibid., p. 38.
20. Ibid., p. 39.
21. Sade dit son admiration pour l'énergie des romans gothiques d'Anne Radcliffe.
22. Jean Sgard souligne l'importance de Prévost pour Sade : «Il est à la fois pour Sade l'idéal inaccessible, le modèle canonique et le matériel inépuisable de toute création romanesque » (Jean Sgard, « De Prévost à Sade, Eighteenth Century Fiction, vol. 1, $\mathrm{n}^{\circ}$ 1, 1988, p. 1-11, p. 1). 
de l'« Idée sur les romans ». En opposant ainsi deux écritures romanesques, Sade se place toujours dans un cadre burkien : comme l'a souligné Terry Eagleton, alors que chez Burke le « beau » est en lien avec le féminin et avec la sociabilité, le « sublime » est clairement du côté du masculin et de la transgression :

Les conditions mêmes qui garantissent l'ordre social sont aussi celles qui le paralysent : absorbés dans cet enfermement narcissique, les hommes de pouvoir se féminisent et perdent leur énergie, et la beauté devient synonyme de stagnation. En réponse à cela, une énergie autre est nécessaire, que Burke trouve dans la tension vers l'effort viril du sublime ${ }^{23}$.

L'opposition entre ces deux écritures est éclairante pour saisir la poétique des Crimes de l'amour, et, tout particulièrement ici, d'« Eugénie de Franval ». Alors que la production ésotérique de Sade, avec ses spectacles répétés à l'infini de la vertu humiliée et détruite, avec ses personnages monstrueux et démesurés, s'inscrit résolument dans la veine sublime, il semble qu'il se soit livré, dans certaines de ses œuvres exotériques, à une tentative d'hybridation, en introduisant dans cette veine des éléments galants et sentimentaux.

Le personnage de Franval est caractéristique de l'ambivalence de l'ensemble du texte. Du héros sadien, il possède de nombreux traits, mais c'est aussi un personnage galant :

Ses vices [...] étaient bien plutôt les torts de l'âge mûr que les inconséquences de la jeunesse [...] de l'art, de l'intrigue... de la méchanceté, de la noirceur, de l'égoïsme, beaucoup de politique, de fourberie, et gazant tout cela, non seulement par les grâces et les talents dont nous avons parlé, mais même par l'éloquence, par infiniment d'esprit, et par les dehors les plus séduisants ${ }^{24}$.

La noirceur du personnage est dissimulée, « gazée » par des attributs prisés dans les cercles mondains : « les grâces », « l'éloquence ", l' « esprit ». On retrouve le terme de "gaze » plus loin, lorsque Sade évoque les projets de Franval à l'égard de sa fille : «[...] se contentant d'une légère gaze, simplement à cause du public, il marcha droit à son horrible but ${ }^{25} »$; et quand l'exhibition du corps d'Eugénie organisée par le libertin pour satisfaire son ami Valmont s'achève, c'est une « gaze $^{26} »$ qui tombe. L'emploi

23. «The very conditions which guarantee social order also paralyse it: sunk in this narcissitic closure, men of affairs grow effete and enervated, sympathy becomes cloying and incestuous, and beauty sinks into a byword for stagnation. Some countervailing energy is therefore necessary, which Burke discovers in the virile strenuousness of the sublime ». (Terry Eagleton, The Ideology of the Aesthetic, Oxford, Royaume-Uni, Cambridge, Massachussets, États-Unis, Blackwell Publishers, 1990, p. 54). Notre traduction.

24. Sade, «Eugénie de Franval », dans Les Crimes de l'amour, op. cit., p. 291-379, p. 294.

25. Ibid., p. 297.

26. Ibid., p. 349. 
du terme "gaze », caractéristique de l'esthétique des romans libertins galants, est à souligner. Dans sa préface, Michel Delon note que « la gaze, c'est aussi l'euphémisme du langage classique ${ }^{27} »$. Ce qui est proposé ici, c'est un texte sadien « gazé », dans lequel on retrouve l'univers de l'auteur de Justine, mais un univers mis à distance, grâce à la recherche dans la veine galante d'une langue, de thèmes et des topoï destinés à masquer la violence du propos.

Métaphores, implicite, ellipses : Sade a recours à ces procédés pour jeter un voile de "gaze» sur ses passages les plus licencieux, comme l'avaient fait avant lui des romanciers libertins galants. La scène d'inceste, qu'on trouve initialement dans le manuscrit des Crimes de l'amour, est évoquée de manière elliptique dans la version publiée - « Telle est l'époque où Franval voulait consommer son crime : Frémissons!... Il le fut ${ }^{28} \gg$. De la même façon, l'auteur supprime dans le texte imprimé la description de la mise en scène imaginée par le libertin pour offrir le corps de sa fille aux regards de son complice Valmont. Si la nouvelle tragique et le roman gothique $^{29}$ sont des modèles qui ont manifestement inspiré le Sade des Crimes de l'amour, le lieu qu'a préparé Franval pour son union avec sa fille rappelle la topographie des «petites maisons » décrites par Bastide, La Morlière ou Crébillon :

Le jour même qu'elle arrive à cet âge, ou plutôt celui qu'il est révolu, se trouvant tous deux à la campagne, sans parents et sans importuns, le comte, après avoir fait parer ce jour-là sa fille comme ces vierges qu'on consacrait jadis au temple de Vénus, la fit entrer, sur les onze heures du matin, dans un salon voluptueux dont les jours étaient adoucis par des gazes, et dont les meubles étaient jonchés de fleurs. Un trône de roses s'élevait au milieu; Franval y conduit sa fille ${ }^{30}$.

L'isolement à la campagne, l'allusion au «temple de Vénus », les perceptions altérées, les gazes, le demi-jour, la confusion entre le naturel et l'artificiel font du lieu un décor caractéristique de l'esthétique galante. Nombreux sont par ailleurs les ressorts narratifs empruntés à la tradition des récits galants dans « Eugénie de Franval »-lettres contrefaites, effraction, correspondances privées révélées, tentatives de séduction, manipulation des domestiques...

C'est cependant l'énergie du sublime qui intéresse Sade dans sa nouvelle. Avec Franval, il tente de retrouver la démesure d'un Lovelace, de manière à

27. Michel Delon, « Préface », dans Sade, Les Crimes de l'amour, op. cit., p. 14.

28. Sade, «Eugénie de Franval », op. cit., p. 302.

29. Sur l'influence du roman gothique sur la poétique de Sade, voir notamment : Annie Le Brun, Les Châteaux de la subversion, Paris, Gallimard, 1986; Stéphanie Genand, Le Libertinage et l'histoire : politique de la séduction à la fin de l'Ancien Régime, Oxford, Voltaire Foundation, coll. « SVEC », n 11, p. 135-142.

30. Sade, « Eugénie de Franval », op. cit., p. 302. 
fasciner son lecteur tout en l'effrayant. Du héros de Richardson, il a la perfection physique, la violence des emportements et la scélératesse revendiquée. Tout comme lui, c'est un comédien accompli, comme en témoigne la fausse scène de réconciliation qu'il organise pour son épouse, pour la convaincre de le suivre dans ses terres de Valmor, où il doit se réfugier après le meurtre de Valmont. D'emblée, Sade souligne le « sang-froid ${ }^{31}$ » du personnage, au moment même où il se lance dans une tirade pathétique. Comme le comédien du Paradoxe de Diderot, c'est parce qu'il joue de tête, parce qu'il maîtrise ses gestes et ses expressions, qu'il réussit à convaincre son public - ici, sa malheureuse épouse - de l'authenticité de ce qu'il exprime. Pour traduire son trouble et son émotion, il a recours à une syntaxe heurtée dans un discours où les interruptions et les exclamations se succèdent, discours combiné avec une rhétorique corporelle - les larmes, l'agenouillement - qui achève de persuader sa victime. Tant d'habileté déployée pour servir un aussi noir dessein - Franval a l'intention de faire assassiner son épouse par sa propre fille -, voilà qui est propre à susciter l'effroi du lecteur.

Sade propose au lecteur plusieurs tableaux pathétiques de la détresse de Madame de Franval, de manière à faire naître en lui le sentiment du sublime. Bien sûr, l'évocation des tourments d'une héroïne en proie aux affres de la passion est déjà un thème majeur de la tradition galante, comme en témoignent les œuvres de Racine, de Guilleragues, ou de la Présidente Ferrand. Cependant, le système de représentation choisi dans « Eugénie de Franval » marque une prédilection pour l'esthétique du sublime qui s'impose au tournant des Lumières. Il ne s'agit pas d'émouvoir le lecteur avec l'évocation délicate des tourments sentimentaux du personnage, comme dans les textes galants, mais bien de le bouleverser par une peinture des effets physiques de la passion, grâce à un dispositif pictural qui privilégie l'expression paroxystique de la violence des souffrances du personnage. Le romancier souligne la composante esthétique du premier tableau présentant le désespoir de Mme de Franval, après qu'elle a été abandonnée par son mari, en comparant la jeune femme aux vierges éplorées de douleur de Michel-Ange :

Quelle victime, hélas! Mme de Franval, pour lors âgée de trente et un ans, était à la fleur de sa plus grande beauté; une impression de tristesse, inévitable d'après les chagrins qui la consumaient, la rendait plus intéressante encore; inondée de larmes, dans l'abattement de la mélancolie... ses beaux cheveux négligemment épars sur une gorge d'albâtre... ses lèvres amoureusement empreintes sur le portrait chéri de son infidèle et de son tyran, elle ressemblait à ces belles vierges que peignit Michel-Ange au sein de la douleur ${ }^{32}$.

Burke l'a bien souligné, «la beauté souffrante est la plus touchante des beautés ${ }^{33} »$. Ici, les souffrances du personnage sont traduites par une 
rhétorique uniquement corporelle - les larmes, l'abattement - teintée d'érotisme - les cheveux « épars sur une gorge d'albâtre 》-, caractéristique de l'esthétique du tableau qui tend à s'imposer dans les romans de la seconde moitié du XVIII ${ }^{\mathrm{e}}$ siècle ${ }^{34}$. On retrouve un pareil tableau lorsque Franval révèle à son épouse les fausses lettres destinées à convaincre le public de sa liaison alléguée avec Valmont. Horrifiée par tant d'abjection, « elle était à genoux, [...] ses mains saignantes et blessées du fer nu dont elle s'efforçait de se saisir pour déchirer son sein [...]»; le sein découvert, les « cheveux en désordre », le visage inondé de larmes, le sang : autant d'éléments censés bouleverser le lecteur, comme le souligne le narrateur : «Jamais la douleur n'eut plus de pathétique et plus d'expression, jamais on ne l'avait vue sous les détails plus touchants, plus intéressants et plus nobles ${ }^{35} »$. Ce tableau est perçu avec indifférence par Franval, qui, à l'issue de cette scène, laisse sa femme évanouie et aux portes de la mort. Le sublime de la confrontation repose à la fois sur la souffrance exhibée de l'épouse, poussée à son paroxysme physique, et sur la monstrueuse et terrifiante indifférence du mari.

Ayant identifié deux traditions dans l'histoire du genre romanesque, l'une galante et sentimentale, liée au féminin, l'autre sublime et clairement marquée du côté du masculin, Sade s'emploie à les fusionner dans le corps du texte d' «Eugénie de Franval». En peignant la violence des passions humaines, Sade propose au lecteur une expérience du sublime débarrassée de tout caractère religieux, un sublime purement humain; mais alors que dans les œuvres ésotériques, cette recherche débouche sur une écriture de l'excès et de la transgression, le romancier s'efforce ici de canaliser son inspiration, par le recours à l'ellipse, à une écriture gazée et à des emprunts à la tradition galante. La question de la nature du geste créateur - jaillissement incontrôlé ou contrainte assumée - est au cœur de l'écriture sadienne dans les nouvelles des Crimes de l'amour. Mais cette question constitue également le thème majeur d'un texte qui met en scène un créateur - Franval dont la déchéance peut se lire comme une absorption dans le féminin.

En peignant la relation entre Franval et Eugénie, Sade propose une réécriture du mythe de Pygmalion ${ }^{36}$, ce qui lui permet de développer une réflexion sur la nature de l'acte de création, réflexion qui repose en définitive sur une «théorie des genres ». Pour le romancier, le jaillissement

34. Sur les liens entre l'esthétique du sentiment et le tableau dans les récits du XVIII siècle, voir notamment: David Denby, Sentimental Narrative and the Social Order in France, 1760-1820, Cambridge, Cambridge University Press, 1994; Anne Coudreuse, Le Goût des larmes au XVIII siècle, Paris, PUF, 1999, rééd. Paris, Desjonquères, 2013.

35. Sade, «Eugénie de Franval », op. cit., p. 331.

36. Sur ce sujet, voir: Aurélia Gaillard, «Aimer une statue : Pygmalion ou la fable de l'amour comblé », Intermédialités : histoire et théorie des arts, des lettres et des techniques, $\mathrm{n}^{0}$ 4, 2004, p. 67-85; dans ce volume, Chiara Gambacorti, «Un Pygmalion des Lumières? Inceste et émancipation féminine dans "Eugénie de Franval" », p. 113-124. 
tumultueux de l'inspiration a clairement à voir avec une énergie liée au sublime et située du côté du masculin. Telle est la conception qu'il développe dans son «Idée sur les romans », lorsqu'il s'interroge sur « les règles de l'art d'écrire un roman ${ }^{37} »$. L'acte d'écrire est associé à l'acte sexuel, à la transgression et à un déferlement de l'imagination :

\footnotetext{
Ô toi qui veux parcourir cette épineuse carrière! ne perds pas de vue que le romancier est l'homme de la nature; elle l'a créé pour être son peintre; s'il ne devient pas l'amant de sa mère dès que celle-ci l'a mis au monde, qu'il n'écrive jamais, nous ne le lirons point; mais s'il éprouve cette soif ardente de tout peindre, s'il entrouvre avec frémissement le sein de la nature, pour y chercher son art et pour y puiser des modèles, s'il a la fièvre du talent et l'enthousiasme du génie, qu'il suive la main qui le conduit, il a deviné l'homme, il le peindra; maîtrisé par son imagination, qu'il y cède, qu'il embellisse ce qu'il voit $[\ldots]^{38}$.
}

« Fièvre », « enthousiasme »: le romancier capte les puissances telluriques de la nature, de manière à la fois active - il doit devenir « l'amant de sa mère »- et passive - il doit céder à son imagination, se laisser dominer par elle. L'image de l'inceste renvoie à l'énergie sexuelle qui se trouve libérée dans l'acte d'écriture, et à la pratique nécessaire de la transgression des codes sociaux, d'où les multiples images de débordement dans le texte de l'«Idée ». Le créateur inspiré par la nature ne doit être contenu «par aucune digue », il lui faut suivre ses «élans », dépasser ses « plans ${ }^{39} »$. Pour Sade, cette nature est « grande, quand elle peuple la terre d'Antonins et de Titus; affreuse, quand elle y vomit des Andronics ou des Nérons; mais toujours sublime ${ }^{40} \gg$. La recherche du sublime, y compris à travers la représentation de spectacles et de personnages terribles, voilà ce qui doit préoccuper le romancier, à l'exclusion de toute visée morale. Le conseil est donné explicitement : «Évite l'afféterie de la morale ${ }^{41} »$. Significativement, le terme péjoratif d' ' afféterie » est associé dans les dictionnaires de l'époque au féminin. Dans la quatrième édition du Dictionnaire de l'Académie française $(1762)^{42}$, l'afféterie est désignée comme une " manière affétée de parler, ou d'agir par envie de plaire » tandis que l'exemple est parlant : « Il y a trop d'afféterie en tout ce qu'elle fait. Les afféteries d'une coquette, d'une précieuse ». Jean-François Féraud souligne cette association au féminin, dans son Dictionnaire critique de la langue française $(1787-1788)^{43}$ lorsqu'il définit «affecté », l'adjectif dont le nom est

37. Sade, «Idée sur les romans », op. cit., p. 42.

38. Ibid., p. 44.

39. Ibid., p. 45.

40. Ibid., p. 47.

41. Ibid., p. 46.

42. Dictionnaire de l'Académie française, $4^{e}$ édition, 2 vol., Paris, chez la Veuve B. Brunet, 1762.

43. Jean-François Féraud, Dictionnaire critique de la langue française, 3 vol., Marseille, Mossy, 1787-1788. 
dérivé : «Plein ou pleine d'affectation. Il ne se dit que d'une femme ou d'une fille coquette ». Le rapport au féminin dans l'acte de création est cependant ambivalent. Si la littérature moralisante, et de manière indirecte la tradition galante, préoccupée d'analyses psychologiques subtiles, de raffinements et de mesure, se trouvent condamnées, il ne faut pas oublier que c'est à la mère nature que doit s'unir l'écrivain, et que le féminin est aussi à l'origine de l'inspiration du créateur.

Le personnage de Franval correspond par bien des aspects au portrait du romancier que Sade appelle de ses vœux dans son « Idée ", jusque dans son rapport complexe au féminin. " Un désordre d'imagination, au-delà de tout ce qu'on peut peindre, était le premier défaut de Franval », affirme l'écrivain, qui poursuit en soulignant qu'avec l'âge, « moins on agit, plus on invente $»$. À cette puissance d'imagination s'ajoute comme chez le créateur sadien le «mépris pour les devoirs moraux et religieux ${ }^{44}$ ». Par ailleurs, agissant en véritable Pygmalion, il fait de sa fille Eugénie sa création, l'image de l'inceste entre le père et la fille venant se surimposer à celle du romancier s'unissant à sa mère la nature ${ }^{45}$. Créateur d'un personnage et concepteur des intrigues du récit, il apparaît comme un double de l'auteur ${ }^{46}$.

Cependant, la confusion générique, caractéristique de la poétique d'« Eugénie de Franval »-tension entre l'énergie du sublime et l'apport de la tradition sentimentale et galante - fait de Franval un personnage singulier. En séduisant sa fille, Franval a établi avec elle une relation d'emprise caractéristique $^{47}$ : «Elle ne voyait que Franval au monde; elle n'y distinguait que lui, elle se révoltait à l'idée de tout ce qui aurait pu l'en séparer ${ }^{48}$ ". Mais cette relation d'emprise est aussi une relation de dépendance mutuelle,

44. Sade, «Eugénie de Franval », op. cit., p. 292.

45. Les liens entre inceste et création dans «Eugénie de Franval» avaient déjà été soulignés par Georges Benrekassa : «Non seulement il est une pratique sociale aristocratique qui trangresse une loi humaine prétendue universelle, et vise à la destruction délibérée de la famille, qui est l'institution par excellence propre à perpétuer l'illusion du naturel dans le social, mais aussi il permet de réaliser une figure privilégiée : celle de l'homme s'exaltant et s'adorant dans sa propre création aux dépens de la création dite naturelle » (Georges Benrekassa, « Loi naturelle et loi civile : l'idéologie des Lumières et la prohibition de l'inceste », Studies on Voltaire and the Eighteenth Century, no 87, 1972, p. 141).

46. Ce personnage complexe, en plus d'être un double du romancier, est aussi une figure de philosophe, comme le remarque Christophe Martin : «Parfaitement indifférent, en effet, aux "préjugés", Franval cumule de nombreux traits faisant de lui une remarquable figure de philosophe » (Christophe Martin, "Éducations négatives ». Fictions d'expérimentation pédagogique négatives au dix-huitième siècle, Paris, Classiques Garnier, 2010, p. 243).

47. Dans Éducations négatives, Christophe Martin montre que cette relation d'emprise est l'aboutissement d'un dispositif pédagogique fortement inspiré par une lecture du livre 5 de l'Émile de Rousseau : « [D]ans le plan d'éducation suivi par Franval, les allusions à la pédagogie rousseauiste paraissent, pour le coup, assez transparentes » (Ibid., p. 274).

48. Sade, «Eugénie de Franval », op. cit., p. 300. 
puisqu'un amour authentique lie les deux personnages ${ }^{49}$. Franval l'avoue à Clervil : « Oui, monsieur, j'aime ma fille, je l'aime avec passion, elle est ma maîtresse, ma femme, ma sœur, ma confidente, mon amie, mon unique dieu sur la terre ${ }^{50}[\ldots] »$. La soumission revendiquée peut se lire comme un désir d'absorption dans le féminin et apparaît comme un aveu de faiblesse. Les grands libertins de Sade s'imposent une ascèse, des exercices d'insensibilité qui leur permettent de composer ces tableaux qu'on trouve dans les œuvres ésotériques et qui tendent vers un sublime parfaitement déshumanisé. Or Franval amoureux est incapable de cette ascèse. Au lieu de catalyser les forces de la nature par une tension énergique vers le sublime, il se laisse dominer par celles-ci. Alors que le héros sadien tend vers une autonomie subjective totale, le désespoir et la faiblesse de Franval, à la fin du récit, sont caractéristiques d'un personnage dépendant et anéanti par la disparition de sa muse. Le tableau du personnage aux prises avec la tempête vient allégoriser cette déchéance du créateur devenu impuissant. Dépouillé de sa fortune et de ses habits par des brigands, «Franval, à moitié nu » tient « toujours son épée 》- ultime et dérisoire attribut de sa puissance passée - et s'effondre : "Épuisé de fatigue, anéanti par la douleur... dévoré d'inquiétude, tourmenté de la tempête, il se jette à terre, et là, les premières larmes qu'il ait versées de sa vie viennent par flots inonder ses yeux ${ }^{51} »$. Le statut esthétique de Franval s'est modifié : lui qui se voulait démiurge, qui avait fait de sa fille sa création et qui contemplait avec indifférence les tableaux de la souffrance de son épouse, se trouve absorbé dans le tableau pathétique de son propre désespoir. Significativement, les deux images utilisées par Sade pour décrire l'acte de création romanesque sont présentes ici, mais leur symbolique se trouve inversée : alors que la fusion avec la mère nature - à travers l'image de l'inceste - était le préalable à la libération de la puissance créatrice, le tableau de Franval à terre renvoie au contraire à la faiblesse d'un personnage terrassé par des forces qui le dépassent; et aux multiples images de débordement que l'on trouve dans l'« Idée »- et qui traduisaient l'énergie créatrice - correspond le déferlement des larmes du personnage brisé par l'émotion. Cette impuissance, c'est aussi celle d'un spectateur. Alors que Franval était à l'origine des grands tableaux sublimes du désespoir de son épouse, c'est à Clervil qu'il revient de relater le plus terrible, et, partant, le plus sublime de ces tableaux, celui de l'agonie de la mère et du suicide de la fille matricide. La réaction de Franval est révélatrice : " Clervil se tait. Il jette ses regards sur Franval; il le voit pétrifié par la douleur; ses yeux étaient fixes, il en coulait des larmes,

49. Aurélia Gaillard remarque que dans « Eugénie de Franval », l'inceste « est décrit non comme une "déviance" du comportement amoureux mais bien comme le comble de l'amour comblé » (Aurélia Gaillard, Pygmalion ou la fable de l'amour comblé, op. cit., p. 84).

50. Sade, « Eugénie de Franval », op. cit., p. 338.

51. Ibid., p. 370. 
mais aucune expression ne pouvait arriver sur ses lèvres ${ }^{52} \gg$. Le créateur énergique n'est plus qu'un sujet pathétique ${ }^{53}$.

Tout acte de création suppose l'établissement de cette relation ambivalente au féminin : telle semble être la leçon de Sade dans 1'" Idée sur les romans » et dans « Eugénie de Franval ». Qu'il s'agisse des forces de la mère nature à laquelle le romancier est sommé de s'unir, ou de la fusion amoureuse des corps et des consciences que rend possible la double relation d'emprise incestueuse qui lie le père à sa fille dans le récit, l'acte de création suppose une absorption dans le féminin, en un mouvement qu'il est cependant impératif de dépasser par une recherche du sublime, qui suppose à la fois une ascèse et une énergie propre à dominer des forces aussi puissantes que destructrices, la déchéance de Franval le montre bien. On pourrait lire cette déchéance comme une concession à la morale du temps, mais elle nous apparaît comme inscrite dans la logique à la fois poétique et narrative $\mathrm{du}$ texte, et on peut lire la trajectoire de Franval comme une allégorie des difficultés du projet de Sade dans Les Crimes de l'amour. La confusion des genres - les traditions romanesques tout autant que les identités sexuelles -, nécessaire à l'acte de création, menace d'anéantir le créateur si elle n'est pas transcendée par une tension constante vers le sublime.

\author{
Dominique Hölzle \\ Université Stendhal - Grenoble 3, LIRE
}

52. Ibid., p. 376.

53. Chiara Gambacorti considère que la punition de Franval dans ce dénouement est artificielle, et doit inciter le lecteur des Crimes de l'amour à se défier des prétentions moralisantes du texte, afin qu'il se livre à une lecture distanciée de récits qu'elle considère comme aussi subversifs, sinon plus, que les œuvres ésotériques: "L'incestueux protagoniste d'"Eugénie de Franval", en effet, se tue in fine sur le corps de sa femme tourmenté par le remords, alors qu'il n'a jamais montré pour elle que du mépris et de la haine. D'ailleurs sa mort, plus qu'une expiation des fautes commises envers elle, apparaît plutôt comme une ultime profanation » (Chiara Gambacorti, « “...CES TABLEAUX DU CRIME TRIOMPHANT...", Écriture moralisante et perversion textuelle dans Les Crimes de l'amour du Marquis de Sade », Dix-huitième siècle, n 39, 2007, p. 547). 